\title{
Determination of Triclinic Bravais Lattice by Means of EBSD Pattern
}

\author{
Ming Han ${ }^{1}$, Guangming Zhao ${ }^{1}$ \\ 1. School of Materials Science and Engineering, East China Jiaotong University, Nanchang, Jiangxi, \\ China
}

Conventionally, the determination of triclinic crystal structures is accomplished by X-ray or electron diffraction techniques together with subsequent structure refinement. Recently, a new technique for determining unknown Bravais lattices based on electron backscatter diffraction (EBSD) pattern has been successfully developed $[1,2]$. In this paper, the advantage of this technique is demonstrated. As far as we know, for the first time, the triclinic Bravais lattice of unknown bulk crystalline materials is directly determined using a single EBSD pattern.

The crystals of albite and wollastonite were chosen for intensity simulation by means of a software DynamicS (Bruker-Nano) [3]. The simulated EBSD pattern of albite $\left(a_{1}=0.8290 \mathrm{~nm}, b_{1}=1.2966 \mathrm{~nm}, c_{1}\right.$ $\left.=0.7151 \mathrm{~nm}, \alpha_{1}=91.18^{\circ}, \beta_{1}=116.31^{\circ}, \gamma_{1}=90.14^{\circ}[4]\right)$ was then treated as a pattern originated from an unknown crystal and used to determine its Bravais lattice, as shown in Figure 1a. The pattern is overlaid by blue lines (marking the virtual traces of diffracting lattice planes) and red circles (representing the zone axes). The red hyperbola segments located in the four corners of Figure 1a are calculated after determining the pattern, which show a convincing correlation to the corresponding edges of the simulated Kikuchi bands, and hence indicating that the traces (blue lines) and zone axes (red circles) have been correctly aligned for the lattice determination. It is interesting to note that the derived Bravais lattice belongs to the primitive triclinic (with $a_{2}=0.7208 \mathrm{~nm}, b_{2}=0.7752 \mathrm{~nm}, c_{2}=0.7770 \mathrm{~nm}, \alpha_{2}=114.68^{\circ}, \beta_{2}=102.99^{\circ}, \gamma_{2}$ $=104.75^{\circ}$ ), which is quite different from that used for the pattern simulation. The reason is explained as follows.

Figure $1 \mathrm{~b}$ presents the atomic arrangement of the crystal albite, viewed along its $c_{1}$ axis. There is a correspondence between the two sets of basis vectors $\left(\boldsymbol{a}_{1}, \boldsymbol{b}_{1}, \boldsymbol{c}_{1}\right.$ and $\left.\boldsymbol{a}_{2}, \boldsymbol{b}_{2}, \boldsymbol{c}_{2}\right)$, cf. the red and blue background, respectively. It can be found that

$a_{2}=-c_{1}, b_{2}=-0.5 a_{1}-0.5 b_{1}, c_{2}=-0.5 a_{1}+0.5 b_{1}$

Inversely,

$a_{1}=-b_{2}-c_{2}, b_{1}=-b_{2}+c_{2}, c_{1}=-a_{2}$

According to the published crystal structure and Eqs. (1) and (2), the parameters of the blue unit cell can be calculated to be $a_{2}=0.7151 \mathrm{~nm}, b_{2}=0.7686 \mathrm{~nm}, c_{2}=0.7703 \mathrm{~nm}, \alpha_{2}=114.81^{\circ}, \beta_{2}=102.78^{\circ}, \gamma_{2}=$ $104.86^{\circ}$, which are quite close to the determined parameters based on Figure 1a. Moreover, all atomic relative coordinates (with respect to $\boldsymbol{a}_{\mathbf{1}}, \boldsymbol{b}_{\mathbf{1}}, \boldsymbol{c}_{\mathbf{1}}$ ) in the published structure (red background) can be translated into the unit cell (defined by $\boldsymbol{a}_{2}, \boldsymbol{b}_{2}, \boldsymbol{c}_{2}$ ) and exactly superpose those atoms inside the unit cell with blue background. This result strongly suggests that the determined unit cell in the present paper can correctly describe the crystal structure of albite. 
In fact, the unit cell defined by the basis vectors $\boldsymbol{a}_{\mathbf{1}}, \boldsymbol{b}_{\mathbf{1}}, \boldsymbol{c}_{\mathbf{1}}$ is actually "base-centered triclinic" which does not exist in the 14 Bravais lattices. During the 3-dimentional reconstruction, both the reduction treatment from a primitive cell to one of the Niggli cells and the corresponding reduction algorithm can only result in a primitive triclinic Bravais lattice, rather than the non-primitive triclinic [5]. Moreover, the parameters of a triclinic unit cell should be conventionally specified to obey the requirement of $a<b<c$. As a result, it is apparent that the primitive triclinic cell of the crystal albite can be correctly recognized by means of a single EBSD pattern [6].

\section{References:}

[1] L. Li, S. Ouyang, Y. Yang, M. Han, J. Appl. Cryst. 47 (2014), p. 1466.

[2] L. Li, M. Han, J. Appl. Cryst. 48 (2015), p. 107.

[3] A. Winkelmann, C. Trager-Cowan, F. Sweeney, A.P. Day, P. Parbrook, Ultramicroscopy 107 (2007), p. 414.

[4] G.E. Harlow, Am. Mineral. 67 (1982), p. 975.

[5] P. Niggli, Krystallographische und strukturtheoretische Grundbegriffe, Handbuch der Experimentalphysik, Akademische Verlagsgesellschaft, Leipzig, 1928.

[6] This work is supported by the Natural Science Foundation of China under grant No. 51071125, and Major Project of Natural Science Foundation of Jiangxi Province under grant No. 20161ACB20010.
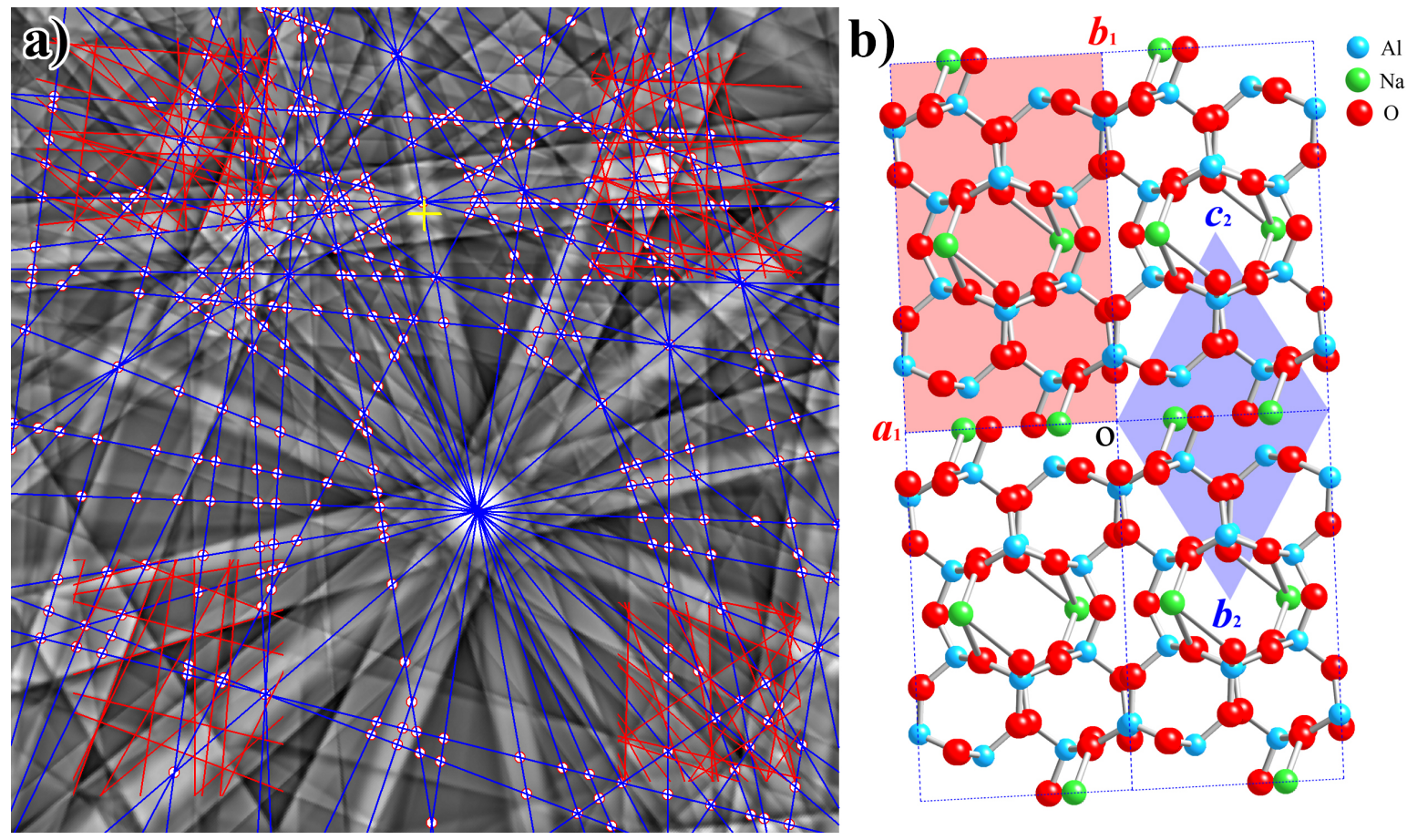

Figure 1. EBSD pattern and crystal structure of albite. a) Simulated pattern of crystal albite overlaid by the virtual traces of diffracting lattice planes (blue lines) and the zone axes (red circles). The detector distance and accelerating voltage used in the simulation is 1.0 and $10 \mathrm{kV}$, respectively. The yellow cross marks the pattern center. b) Arrangement of the atoms in crystal albite viewed along $\boldsymbol{c}_{1}$ or $\boldsymbol{a}_{2}$ axis. The red background defined by $\boldsymbol{a}_{1}$ and $\boldsymbol{b}_{1}$ axes indicates the reported unit cell in previous literatures, while the blue bordered by $\boldsymbol{b}_{2}$ and $\boldsymbol{c}_{2}$ axes is the unit cell determined by the EBSD pattern. 